\title{
In vitro antibacterial activity of thymol and carvacrol and their effects on broiler chickens challenged with Clostridium perfringens
}

\author{
Encun Du, Liping Gan, Zhui Li, Weiwei Wang, Dan Liu and Yuming Guo*
}

\begin{abstract}
Background: In the post-antibiotic era, essential oils (EO) are promising alternatives to growth-promoting antibiotics. The aim of the present study was to investigate the antibacterial activities of an EO product and its components thymol and carvacrol in vitro, and the efficacy of EO to control Clostridium perfringens challenge in broiler chickens.

Results: The in vitro minimum inhibitory concentration assay showed strong antibacterial activity of the EO product, thymol, and carvacrol against pathogenic Escherichia coli, C. perfringens, and Salmonella strains, and weak activity towards beneficial Lactobacillus strains. Besides, an additive effect was observed between thymol and carvacrol. The in vivo study was carried out with 448 male broiler chicks following a $4 \times 2$ factorial arrangement to test the effects of EO supplementation $(0,60,120$, or $240 \mathrm{mg} / \mathrm{kg}$ EO in wheat-based diet), pathogen challenge (with or without oral gavage of C. perfringens from day 14 to day 20) and their interactions. Each treatment consisted of eight replicate pens (seven birds/pen). The challenge led to macroscopic gut lesions, and resulted in a significant increase in ileal populations of $C$. perfringens and Escherichia subgroup ( $P \leq 0.05$ ) on day 21. Dietary EO supplementation did not influence $C$. perfringens numbers, but linearly alleviated intestinal lesions on day 21 and 28 ( $P=0.010$ and 0.036 , respectively), and decreased Escherichia populations in ileum with increased EO dosages ( $P=0.027$ and 0.071 for day 21 and 28 , respectively). For caecum, EO quadratically influenced Lactobacillus populations on day $21(P=0.002)$, and linearly decreased the numbers of total bacteria and Escherichia on day $28(P=0.026$ and 0.060 , respectively). Mean thymol and carvacrol concentrations in the small intestine were 0.21 and $0.20 \mu \mathrm{g} / \mathrm{g}$ in intestinal digesta (wet weight), respectively, for birds fed $60 \mathrm{mg} / \mathrm{kg}$ EO, and 0.80 and $0.71 \mu \mathrm{g} / \mathrm{g}$, respectively, for birds fed $240 \mathrm{mg} / \mathrm{kg}$ EO.
\end{abstract}

Conclusions: These results indicated that dietary EO supplementation could affect intestinal microbiota and alleviate intestinal lesions in broilers, which may contribute in controlling C. perfringens infection in broiler chickens.

Keywords: Thymol, Carvacrol, Essential oils, Clostridium perfringens, Escherichia coli, Broiler chickens

\section{Background}

Clostridium perfringens is a Gram-positive, anaerobic, spore-forming bacterium, which is usually classified into five toxinotypes (A, B, C, D, and E) according to the production of four major toxins, called alpha, beta, epsilon and iota. Although $C$. perfringens is a commensal bacterium of the intestine, $C$. perfringens type A is considered the main causative agent of necrotic enteritis (NE) in

\footnotetext{
* Correspondence: guoyum@cau.edu.cn

State Key Laboratory of Animal Nutrition, College of Animal Science and

Technology, China Agricultural University, Beijing 100193, P. R. China
}

poultry $[1,2]$. Proliferation of pathogenic $C$. perfringens and released toxins, especially NetB toxin, result in NE in poultry $[2,3]$. NE is a widespread disease in poultry, as estimated to cost the international poultry industry approximately two billion US dollars annually [4]. Impaired feed digestion and absorption, reduced growth rate, and mortality are the major reasons for production losses associated with NE $[2,5]$.

The use of in-feed antibiotics has been the main strategy for controlling NE in poultry. However, public concern about the threat of antibiotic-resistant pathogens 
has forced the poultry industry to consider alternatives to antibiotics for poultry production [5]. Probiotics, prebiotics, organic acids, enzymes, and essential oils (EO) are among the alternatives [5]. Also, because of consumer preference for natural products, the use of EO has increased appeal [6].

The antibacterial properties of EO have long been recognized and widely tested in vitro against a wide range of pathogenic bacteria, including both Gram positive and Gram negative bacteria [6-8]. Although the antibacterial mechanism of EO and their constituents is not fully understood, studies have shown that constituents with a phenolic structure, such as eugenol, carvacrol and thymol have the greatest bactericidal activities, followed by aldehydes, ketones, alcohols, ethers and hydrocarbons [9-11]. However, it seems that the efficacy of EO is not consistent in vivo: both improved and unchanged growth performance and intestinal microbiota have been reported in pigs and chickens $[6,8,12-14]$. Actually, it is difficult to compare the efficacy of EO considering the fact that EO blends containing various constituents have been used in vivo in published reports [6, 8, 12-15]. In addition, little information is available regarding the relationship between antibacterial activities in vitro and in vivo. Therefore, the aim of the present study was to investigate the efficacy of an EO product as well as its components thymol and carvacrol on pathogenic bacteria and benefical Lactobacillus strains, and to investigate the effects of EO on broiler chickens challenged with $C$. perfringens. In addition, thymol and carvacrol concentrations in intestinal digesta were assayed.

\section{Methods}

\section{In vitro study}

\section{Chemicals}

Thymol and carvacrol were purchased from SigmaAldrich Corporation (St Louis, MO, USA), with compound purities at $\sim 98 \%$. A commercial EO product was provided by Novus International Inc. (St Charles, MO, USA), which contained $25 \%$ thymol and $25 \%$ carvacrol as active components, $37 \%$ silicon dioxide as a caking inhibitor, and $13 \%$ glycerides as stabilizing agents.

\section{Preparation of cultures}

Thymol, carvacrol and the commercial EO product were individually tested against a panel of undesirable bacteria and beneficial Lactobacillus strains. The undesirable bacteria included two chicken Escherichia coli field strains CVCC1553 and CVCC1490 (serotype O78), chicken C. perfringens field strains (CVCC2027 and CVCC2030), Salmonella enterica serovar Typhimurium (CVCC541), Salmonella enterica serovar Enteritidis (CVCC2184) and Salmonella enterica serovar Pullorum (C79-13), which were obtained from China Veterinary Culture Collection
Center (Beijing, China). Another $S$. Enteritidis field strain (ATCC13076) was obtained from American Type Culture Collection (Manassas, VA, USA). In addition, three strains of Lactobacillus were tested. L. acidophilus (GIM1.730) was obtained from Guangdong Microbiology Culture Center (Guangdong, China), and L. reuteri and $L$. salivarius were isolated from the gastrointestinal content of healthy broiler chickens by our laboratory staff.

Overnight cultures of E. coli and Salmonella were prepared freshly by cultivation from frozen stock at $37^{\circ} \mathrm{C}$ in Luria-Bertani broth with continuous shaking. Cultures of $C$. perfingens were prepared anaerobically at $37{ }^{\circ} \mathrm{C}$ overnight in cooked meat medium and Man-RogosaSharpe (MRS) broth used for growing of Lactobacillus at $37^{\circ} \mathrm{C}$ without shaking.

\section{Minimum inhibitory concentration and minimal bactericidal concentration assay}

The minimum inhibitory concentration (MIC) was determined via a conventional broth dilution method, as described by Ivanovic et al. with some modifications [16]. Briefly, the commercial EO product, thymol, and carvacrol were initially dissolved as $300 \mathrm{mg} / \mathrm{mL}$ stock solution in dimethylsulfoxide (DMSO) and diluted to $6 \mathrm{mg} / \mathrm{mL}$ in Mueller-Hinton broth with vigorous shaking. Then, two-fold serial dilutions of thymol and carvacrol were prepared, producing concentrations at 6000 , 3000, 1500, 750, 375, 187.5, 93.75 and $46.875 \mu \mathrm{g} / \mathrm{mL}$. For the detection of Lactobacillus, MRS broth was used for dilution. In order to avoid the antibacterial effects of DMSO itself, the final DMSO concentrations never exceeded $2 \%$ (by volume). Then, each diluted broth was inoculated with fresh microbial suspension (final concentration: $1 \sim 5 \times 10^{5}$ colony-forming units $\left.(\mathrm{cfu}) / \mathrm{mL}\right)$. E. coli and Salmonella were incubated at $37{ }^{\circ} \mathrm{C}$ with continuous shaking overnight. C. perfringens and Lactobacillus were incubated anaerobically at $37{ }^{\circ} \mathrm{C}$ for 24 and $16 \mathrm{~h}$, respectively, without shaking. A positive growth control containing $2 \%$ DMSO without EO or components, and a negative control containing no bacteria were included in each experiment. After incubation, the optical density (OD) of suspension was measured using a spectrophotometer at $595 \mathrm{~nm}$. In addition, $50 \mu \mathrm{L}$ from each broth dilution was inoculated for enumeration in duplicate onto nutrient agar for E. coli and Salmonella, sulfite-polymyxin-sulfadiazine agar for $C$. perfringens, and MRS agar for Lactobacillus, all by the spread plate method. MIC was defined as the lowest concentration of EO or components that showed no increase in OD following incubation. The minimal bactericidal concentration $(\mathrm{MBC})$ was defined as the lowest concentration of EO or components with which no viable bacteria were detected. All assays were performed in triplicate. 


\section{Combination assay}

A combination assay between thymol and carvacrol was performed by the checkerboard method, as previously described [17]. Two-fold serial dilutions of one component were tested in the presence of serial concentrations of the other component (which did not inhibit bacterial growth alone). The fractional inhibitory concentration (FIC) was calculated as follows: FIC of component A = MIC of component A in combination divided by the MIC of component $\mathrm{A}$ alone, FIC of component B = MIC of component B in combination divided by the MIC of component $\mathrm{B}$ alone, and FIC index $(\mathrm{FICI})=\mathrm{FIC}$ of component $\mathrm{A}+\mathrm{FIC}$ of component B. An FICI $<0.5$ was considered to demonstrate synergy. When an FICI fell between 0.5 and 1.0, it was defined as an additive effect and, between 1.0 and 4.0, it was classified as no interaction. Finally, an FICI $>4.0$ indicated antagonism between the components in a combination.

\section{In vivo study}

\section{Birds, diets, and experimental design}

All experimental procedures were approved by the China Agricultural University Animal Care and Use Committee. A total of 448 one-day-old male broiler chicks (Cobb 500) were used for a 28-day experiment. Chicks were assigned to eight treatments, following a $4 \times 2$ factorial arrangement in a randomized complete block design to evaluate dietary EO supplementation $(0,60,120$, or $240 \mathrm{mg} / \mathrm{kg} \mathrm{EO}$ in wheat-based diet), pathogen challenge (with or without oral gavage of $C$. perfringens from day 14 to 21 ) and their interactions. Each treatment consisted of eight replicate pens (seven birds/pen). The EO used in this trial was the commercial product mentioned above, which contained $25 \%$ thymol and $25 \%$ carvacrol as active components. Chickens were fed starter (day 0-21) and finisher (day 21-28) diets in the form of mash and had access to feed and water ad libitum. Proliferation of $C$. perfringens was promoted by formulating antibiotic-free and coccidiostat-free wheat-based diets. All nutrients were formulated to meet or exceed the feeding standard of China (NY/T 2004) for broilers [18] (Table 1).

\section{Clostridium perfringens challenge and sampling}

C. perfringens challenge was conducted as originally developed by Dahiya et al. [19]. The particular organism, CVCC2027, was a type A field strain, isolated from a clinical case of NE in chickens, which did not carry the NetB gene, as determined by polymerase chain reaction (PCR). Briefly, the organism was cultured anaerobically on tryptose-sulphite-cycloserine agar base at $37{ }^{\circ} \mathrm{C}$ for $18 \mathrm{~h}$, and then aseptically inoculated into cooked meat medium and incubated anaerobically at $37{ }^{\circ} \mathrm{C}$ overnight. All birds in challenged groups were orally gavaged in the crop once per day with $1.0 \mathrm{~mL}$ of actively growing $C$. perfringens culture from day 14 to $20\left(1.0 \times 10^{8} \mathrm{cfu} / \mathrm{mL}\right)$. On day 21 and 28, one bird per replicate was randomly selected and killed by intracardial administration of sodium pentobarbital (30 mg/ $\mathrm{kg}$ body weight) and jugular exsanguination prior to sample collection.

\section{Intestinal lesion score}

The small intestine from each bird was opened and scored blindly on a scale from zero to four as described by Dahiya et al. [19]: $0=$ normal intestinal appearance with no lesion, $0.5=$ severely congested serosa and mesentery engorged with blood, $1=$ thin walled and friable intestines with small red petechiae (>5), $2=$ focal necrotic lesions, $3=$ patches of necrosis (1 to $2 \mathrm{~cm}$-long), and $4=$ diffused necrosis typical of field cases.

\section{Bacteriological examination}

On day 21 and day 28, digesta for bacteriological examination were collected aseptically from ileum (from ileum midpoint to $2 \mathrm{~cm}$ proximal to ileocecal junction) and caecum, and stored at $-80{ }^{\circ} \mathrm{C}$. Bacterial populations were detected by the method of absolute quantitative real-time PCR (RT-PCR), as described by Wise and Siragusa, with some modifications [20]. Genomic DNA was isolated from $200 \mathrm{mg}$ of digesta from ileum and caecum using a commercial kit (QIAamp DNA Stool Mini Kit, Qiagen Inc., Valencia, CA, USA). Extracted DNA was stored at $-20{ }^{\circ} \mathrm{C}$ until analysis.

Standard curves for RT-PCR were prepared using DNA extracted from pure cultures to produce a high concentration of the target DNA by normal PCR amplification. Primer sequences were used in previous studies, which were designed on the basis of 16s rDNA sequences [21-23]. Target groups, primer sequences, amplicon sizes, and references are shown in Table 2 . The targeted Escherichia subgroup contained genera of E. coli, Hafnia alvei and Shigella [22]. E. coli competent cells DH5a (Takara Bio Inc., Japan) were used to create plasmid standards. Firstly, PCR products were purified using a PCR purification kit (Biomed Gene Technologies, Beijing, China), and then cloned into $\mathrm{pCR}^{\circ} 2.1$ using a TA cloning kit (Invitrogen Corporation, Carlsbad, CA, USA), following the manufacturer's protocol. Purified insert-containing plasmids were quantified using a Nanodrop ND-1000 spectrophotometer (Thermo Fisher Scientific Inc., Waltham, MA, USA), and the number of 
Table 1 Diet composition and nutrient levels

\begin{tabular}{|c|c|c|}
\hline Item (\%, unless otherwise indicated) & Starter diets (day 0-21) & Grower diets (day 22-28) \\
\hline \multicolumn{3}{|l|}{ Ingredient } \\
\hline Wheat & 62.75 & 68.5 \\
\hline Soybean meal & 29.61 & 23.72 \\
\hline Soybean oil & 3.40 & 4.00 \\
\hline Dicalcium phosphate & 1.91 & 1.63 \\
\hline Limestone & 1.04 & 0.96 \\
\hline Sodium chloride & 0.35 & 0.35 \\
\hline Choline chloride (50 \%) & 0.25 & 0.25 \\
\hline L-Lysine (99 \%) & 0.25 & 0.24 \\
\hline DL-Methionine (98 \%) & 0.19 & 0.11 \\
\hline Antioxidants & 0.03 & 0.03 \\
\hline Trace mineral premix ${ }^{a}$ & 0.20 & 0.20 \\
\hline Vitamin premix ${ }^{b}$ & 0.02 & 0.02 \\
\hline \multicolumn{3}{|c|}{ Calculated nutrient levels } \\
\hline Metabolizable energy (Mcal/kg) & 2.90 & 2.98 \\
\hline Protein & 21.00 & 19.00 \\
\hline Calcium & 1.00 & 0.90 \\
\hline Available phosphorus & 0.45 & 0.40 \\
\hline Lysine & 1.15 & 1.00 \\
\hline Methionine & 0.50 & 0.40 \\
\hline
\end{tabular}

a The trace mineral premix provided the following (per kilogram of diet): manganese, $100 \mathrm{mg}$; zinc, $75 \mathrm{mg}$; iron, $80 \mathrm{mg}$; copper, $8 \mathrm{mg}$; selenium, $0.25 \mathrm{mg}$; iodine, $0.35 \mathrm{mg}$

b The vitamin premix provided the following (per kilogram of diet): vitamin A, $18750 \mathrm{IU}$; vitamin $\mathrm{D}_{3}, 3750 \mathrm{IU}$; vitamin $\mathrm{E}$, $28 \mathrm{IU}$; vitamin $\mathrm{K}_{3}$, $3.975 \mathrm{mg}$; thiamine mononitrate, $3 \mathrm{mg}$; riboflavin, $9 \mathrm{mg}$; vitamin $\mathrm{B}_{12}, 0.0375 \mathrm{mg}$; d-biotin, $0.150 \mathrm{mg}$; folic acid, $1.875 \mathrm{mg}$; d-calcium pantothenate, $18 \mathrm{mg}$; nicotinic acid, $75 \mathrm{mg}$

target gene copies was calculated by the following formula according to Lee et al. [24]:

$\operatorname{DNA}($ copy $)=\frac{6.02 \times 10^{23}(\mathrm{copy} / \mathrm{mol}) \times \text { DNA amount }(\mathrm{g})}{\text { DNA length }(\mathrm{dp}) \times 660(\mathrm{~g} / \mathrm{mol} / \mathrm{dp})}$

Ten-fold serial dilutions of plasmid DNA were included on each 96-well plate to produce a standard curve. Genomic DNA from ileal samples and cecal samples was used as templates for absolute quantitative RT-PCR with a 7500 fluorescence detection system (Applied Biosystems, Foster City, CA, USA) according to optimized PCR protocols (SYBR-Premix Ex Taq, Takara Bio Inc., Japan). RT-PCR primers were the same as for normal PCR amplification primers (Table 2). Normal distributions were achieved by showing the results in terms of $\log _{10}$ gene copies/g intestinal digesta.

Table 2 16s rDNA real-time PCR primers used to quantify intestinal bacteria

\begin{tabular}{|c|c|c|c|}
\hline Target & Primer sequence $\left(5^{\prime}-3^{\prime}\right)^{a}$ & Amplicon size (bp) & $\overline{\text { Reference }}$ \\
\hline \multirow[t]{2}{*}{ Total bacteria } & F:ACTCCTACGGGAGGCAGCAGT & \multirow[t]{2}{*}{200} & \multirow[t]{2}{*}{ [19] } \\
\hline & R:GTATTACCGCGGCTGCTGGCAC & & \\
\hline \multirow[t]{2}{*}{ Lactobacillus subgroup } & F:AGCAGTAGGGAATCTTCCA & \multirow[t]{2}{*}{341} & \multirow[t]{2}{*}{ [19] } \\
\hline & R:CACCGCTACACATGGAG & & \\
\hline \multirow[t]{2}{*}{ Escherichia subgroup ${ }^{\text {b }}$} & F:GTTAATACCTTTGCTCATTGA & \multirow[t]{2}{*}{340} & \multirow[t]{2}{*}{ [20] } \\
\hline & R:ACCAGGGTATCTAATCCTGT & & \\
\hline \multirow[t]{2}{*}{ Clostridium perfringens } & F:AAAGATGGCATCATCATTCAAC & \multirow[t]{2}{*}{279} & \multirow[t]{2}{*}{ [21] } \\
\hline & R:TACCGTCATTATCTTCCCCAAA & & \\
\hline
\end{tabular}

${ }^{a} \mathrm{~F}$ means forward, $\mathrm{R}$ means reverse

${ }^{\mathrm{b}}$ The targeted Escherichia subgroup contained genera of E. coli, Hafnia alvei and Shigella 


\section{Chemical analysis for thymol and carvacrol}

On day 21, fresh digesta collected from small intestine ( $5 \mathrm{~cm}$ proximal to Meckel's diverticulum) were acidified to $\mathrm{pH}<2$ with $2 \%$ of $6 \mathrm{~mol} \mathrm{H}_{2} \mathrm{SO}_{4}$ per litre to stop fermentation and stored at $-20{ }^{\circ} \mathrm{C}$ pending analysis for thymol and carvacrol. Quantification of thymol and carvacrol in intestinal digesta was performed, as described by Michiels et al. with some modifications [25]. Briefly, $2.0 \mathrm{~g}$ of digesta from each bird were used for extraction, and $4 \mathrm{~mL}$ ethyl ethanoate was added as extraction solvent. All extractions were performed in duplicate. $2.5 \mathrm{mg}$ of 2-isopropylphenol $/ \mathrm{mL}$ of ethyl ethanoate was used as the internal standard. Ethyl ethanoate extracts were combined and reduced to dryness with nitrogen at room temperature. Then, the residue redissolved in ethyl ethanoate was used for gas chromatographic analysis.

\section{Statistical analysis}

Results are given as mean values and pooled standard errors. Data were analyzed using the general linear model procedure of SAS software (SAS Institute Inc., Cary, NC, USA). The main effects of $C$. perfringens challenge, EO supplementation, and their interactive effects were analyzed. Duncan's multiple-range tests were used to separate means when interactions were significant. When interactions were not significant, polynomial contrasts were conducted to determine linear and quadratic responses of the main-effect means (averaged between challenged and unchallenged groups) to dietary EO dosages. Statistical significance was considered at $P \leq 0.05$, and $0.05<P<0.10$ was considered a trend towards significance.

\section{Results}

\section{In vitro antibacterial activity of EO and components}

The antibacterial activities of EO and components (thymol and carvacrol) against selected pathogenic bacteria and beneficial Lactobacillus strains were expressed as
MICs and MBCs (Table 3). Among the eight pathogenic bacteria, E. coli was more sensitive to thymol (MIC and MBC, 187.5 and $375 \mu \mathrm{g} / \mathrm{mL}$, respectively). In contrast, $S$. Enteritidis was more sensitive to carvacrol (MIC and $\mathrm{MBC}, 187.5$ and $750 \mu \mathrm{g} / \mathrm{mL}$, respectively). However, the greatest MIC and MBC were obtained for thymol in the presence of $S$. Enteritidis ( 750 and $1500 \mu \mathrm{g} / \mathrm{mL}$, respectively). Thymol and carvacrol exhibited the same antibacterial activity against $C$. perfringens, $S$. Typhimurium and S. Pullorum (MIC and MBC, 375 and $750 \mu \mathrm{g} / \mathrm{mL}$, respectively). Moreover, the tested EO product showed identical antibacterial activity against all eight pathogenic bacteria (MIC and MBC, 750 and $1500 \mu \mathrm{g} / \mathrm{mL}$, respectively). In addition, antibacterial activity of thymol and carvacrol was noticed against $L$. acidophilus, $L$. reuteri and $L$. salivarius at concentrations higher than for pathogenic bacteria (MIC and MBC, 1500 and $3000 \mu \mathrm{g} / \mathrm{mL}$, respectively). The EO was also observed to inhibit Lactobacillus growth (MIC and $\mathrm{MBC}$, both $3000 \mu \mathrm{g} / \mathrm{mL}$ ). In addition, the FICI towards all tested bacteria fell between 0.5 and 1.0, demonstrating an additive antibacterial effect between thymol and carvacrol.

\section{Intestinal lesion score}

No intestinal lesions were observed in unchallenged birds. In challenged groups, intestinal lesion scores were reduced linearly with increased dietary EO dosages on day 21 and day $28(P=0.010$ and 0.036 , respectively, Fig. 1). Compared with birds fed basal diet, lesion severity in broilers fed $240 \mathrm{mg} / \mathrm{kg}$ EO was reduced significantly on day $21 \quad(P \leq 0.05)$, and lesion severity of broilers fed 120 and $240 \mathrm{mg} / \mathrm{kg}$ EO was reduced significantly on day $28(P \leq 0.05)$.

\section{Quantitation of ileal and cecal microbiota}

$C$. perfringens challenge led to significant increases in ileal populations of Escherichia subgroup $(P<0.001)$ and C. perfringens $(P=0.03)$ on day 1 post-challenge (Table 4$)$.

Table 3 Antibacterial activity of essential oils and components towards selected bacteria

\begin{tabular}{|c|c|c|c|c|c|c|c|}
\hline \multirow[t]{2}{*}{ Bacteria } & \multicolumn{3}{|c|}{ MIC $(\mu \mathrm{g} / \mathrm{mL})$} & \multicolumn{3}{|c|}{$\mathrm{MBC}(\mu \mathrm{g} / \mathrm{mL})$} & \multirow[t]{2}{*}{$\mathrm{FICl}$} \\
\hline & $\overline{\mathrm{EO}}$ & Thymol & $\overline{\text { Carvacrol }}$ & $\overline{\mathrm{EO}}$ & Thymol & $\overline{\text { Carvacrol }}$ & \\
\hline Escherichia coli & 750 & 187.5 & 375 & 1500 & 375 & 750 & 0.5 \\
\hline Clostridium perfringens & 750 & 375 & 375 & 1500 & 750 & 750 & 0.5 \\
\hline Salmonella Typhimurium & 750 & 375 & 375 & 1500 & 750 & 750 & 0.5 \\
\hline Salmonella Enteritidis & 750 & 750 & 187.5 & 1500 & 1500 & 750 & 1.0 \\
\hline Salmonella Pullorum & 750 & 375 & 375 & 1500 & 750 & 750 & 0.5 \\
\hline Lactobacillus acidophilus & 3000 & 1500 & 1500 & 3000 & 3000 & 3000 & 1.0 \\
\hline Lactobacillus reuteri & 3000 & 1500 & 1500 & 3000 & 3000 & 3000 & 1.0 \\
\hline Lactobacillus salivarius & 3000 & 1500 & 1500 & 3000 & 3000 & 3000 & 1.0 \\
\hline
\end{tabular}

MIC, the minimum inhibitory concentration; MBC, the minimal bactericidal concentration; EO, essential oil contained $25 \%$ thymol and $25 \%$ carvacrol as active components; $\mathrm{FICl}$, fractional inhibitory concentration index 


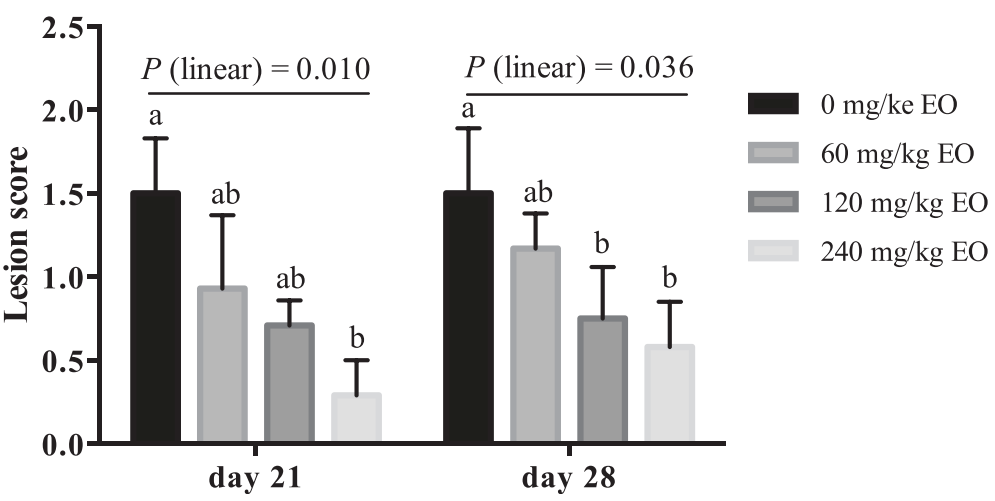

Fig. 1 Effects of essential oils on intestinal lesion score of Clostridium perfringens challenged broiler chickens. Values are means of eight replicates per treatment and pooled standard error of the mean. Bars not sharing a common letter differ significantly $(P \leq 0.05)$. EO, essential oils; $P$ (linear), polynomial contrasts were conducted to determine the linear response of lesion score to dietary EO dosages

However, on day 7 post-challenge, ileal microbiota of challenged birds were restored to the level of unchallenged birds $(P>0.10$, Table 5$)$. Dietary supplementation of EO did not influence $C$. perfringens numbers, but linearly decreased Escherichia numbers in ileum on day 21 and 28 $(P=0.027$ and 0.071 , respectively) regardless of challenge (Tables 4 and 5). For cecal microbiota, the population of $C$. perfringens was increased on day 1 post-challenge $(P<0.001$, Table 6), and showed a tendency to increase on day 7 post-challenge $(P=0.078$, Table 7$)$. Dietary EO supplementation influenced cecal Lactobacillus numbers quadratically $(P=0.002)$ on day 21 (Table 6). Compared with birds fed basal diet, higher Lactobacillus populations were observed in birds fed $60 \mathrm{mg} / \mathrm{kg}$ and $120 \mathrm{mg} / \mathrm{kg}$ EO on day 21 in both unchallenged and challenged groups (Table 6). In contrast, the populations of total bacteria and Escherichia in caecum decreased linearly with increased EO dosages on day $28(P=$ 0.026 and 0.060 , respectively, Table 7 ). No interactions were observed for ileal and cecal microbiota between $C$. perfringens challenge and EO supplementation $(P>0.10$, Table $4,5,6$ and 7$)$.

Table 4 Effect of essential oils on ileal bacterial populations of broilers on day 21

\begin{tabular}{|c|c|c|c|c|}
\hline Item $^{a}$ & Total bacteria & Lactobacillus & Escherichia & C. perfringens \\
\hline \multicolumn{5}{|l|}{ Treatment } \\
\hline Basal diet, unchallenged & 9.00 & 7.89 & 8.00 & 2.27 \\
\hline 60 mg/kg EO, unchallenged & 9.28 & 7.98 & 7.60 & 2.35 \\
\hline 120 mg/kg EO, unchallenged & 9.35 & 8.30 & 7.55 & 2.34 \\
\hline $240 \mathrm{mg} / \mathrm{kg} \mathrm{EO}$, unchallenged & 9.22 & 8.14 & 7.15 & 2.34 \\
\hline Basal diet, challenged & 9.41 & 8.11 & 8.72 & 2.50 \\
\hline 60 mg/kg EO, challenged & 9.48 & 8.38 & 8.99 & 2.39 \\
\hline 120 mg/kg EO, challenged & 9.44 & 8.39 & 8.26 & 2.43 \\
\hline 240 mg/kg EO, challenged & 9.29 & 8.27 & 8.03 & 2.57 \\
\hline SEM & 0.068 & 0.073 & 0.137 & 0.033 \\
\hline \multicolumn{5}{|c|}{$P$-value } \\
\hline Challenge & 0.185 & 0.169 & $<0.001$ & 0.030 \\
\hline EO & 0.735 & 0.438 & 0.095 & 0.805 \\
\hline Challenge $\times \mathrm{EO}$ & 0.828 & 0.888 & 0.713 & 0.655 \\
\hline Linear ${ }^{b}$ & 0.945 & 0.366 & 0.027 & 0.553 \\
\hline Quadratic $^{\text {b }}$ & 0.261 & 0.172 & 0.853 & 0.707 \\
\hline
\end{tabular}

a Values are means of eight replicates per treatment, and expressed as $\log _{10}$ (copy/g digesta). EO, essential oils; unchallenged, birds without challenge of $C$. perfringens; challenged, birds challenged with $C$. perfringens from day 14 to 20; SEM, pooled standard error of the mean; Challenge $\times$ EO, interaction between $C$. perfringens challenge and EO supplementation

${ }^{\mathrm{b}}$ When interaction between C. perfringens challenge and EO supplementation was not significant, linear and quadratic polynomial contrasts were performed on the main-effect means to EO dosages 
Table 5 Effect of essential oils on ileal bacterial populations of broilers on day 28

\begin{tabular}{|c|c|c|c|c|}
\hline Item $^{a}$ & Total bacteria & Lactobacillus & Escherichia & C. perfringens \\
\hline \multicolumn{5}{|l|}{ Treatment } \\
\hline Basal diet, unchallenged & 9.13 & 7.70 & 6.09 & 3.00 \\
\hline $60 \mathrm{mg} / \mathrm{kg} \mathrm{EO}$, unchallenged & 9.08 & 7.76 & 6.27 & 2.79 \\
\hline 120 mg/kg EO, unchallenged & 8.85 & 7.71 & 5.90 & 2.94 \\
\hline 240 mg/kg EO, unchallenged & 9.13 & 7.76 & 5.91 & 2.84 \\
\hline Basal diet, challenged & 9.26 & 7.99 & 6.13 & 2.96 \\
\hline $60 \mathrm{mg} / \mathrm{kg} \mathrm{EO}$, challenged & 9.33 & 8.18 & 6.56 & 3.04 \\
\hline 120 mg/kg EO, challenged & 9.08 & 7.98 & 5.76 & 2.95 \\
\hline $240 \mathrm{mg} / \mathrm{kg}$ EO, challenged & 8.91 & 7.80 & 5.60 & 2.95 \\
\hline SEM & 0.058 & 0.081 & 0.098 & 0.051 \\
\hline \multicolumn{5}{|c|}{$P$-value } \\
\hline Challenge & 0.421 & 0.135 & 0.887 & 0.462 \\
\hline EO & 0.365 & 0.877 & 0.092 & 0.953 \\
\hline Challenge $\times \mathrm{EO}$ & 0.469 & 0.882 & 0.742 & 0.789 \\
\hline Linear ${ }^{b}$ & 0.215 & 0.662 & 0.071 & 0.619 \\
\hline Quadratic $^{b}$ & 0.502 & 0.708 & 0.780 & 0.931 \\
\hline
\end{tabular}

${ }^{a}$ Values are means of eight replicates per treatment, and expressed as $\log _{10}$ (copy/g digesta). EO, essential oils; unchallenged, birds without challenge of $C$. perfringens; challenged, birds challenged with C. perfringens from day 14 to 20; SEM, pooled standard error of the mean; Challenge $\times$ EO, interaction between $C$. perfringens challenge and EO supplementation

${ }^{\mathrm{b}}$ When interaction between C. perfringens challenge and EO supplementation was not significant, linear and quadratic polynomial contrasts were performed on the main-effect means to EO dosages

Table 6 Effect of essential oils on cecal bacterial populations of broilers on day 21

\begin{tabular}{|c|c|c|c|c|}
\hline Item $^{a}$ & Total bacteria & Lactobacillus & Escherichia & C. perfringens \\
\hline \multicolumn{5}{|l|}{ Treatment } \\
\hline Basal diet, unchallenged & 11.16 & 8.52 & 9.77 & 3.05 \\
\hline 60 mg/kg EO, unchallenged & 11.50 & 8.86 & 9.53 & 2.38 \\
\hline 120 mg/kg EO, unchallenged & 11.41 & 9.16 & 9.51 & 2.83 \\
\hline 240 mg/kg EO, unchallenged & 11.42 & 8.69 & 9.41 & 2.41 \\
\hline Basal diet, challenged & 11.28 & 8.60 & 9.28 & 3.68 \\
\hline $60 \mathrm{mg} / \mathrm{kg} \mathrm{EO}$, challenged & 11.43 & 9.02 & 9.42 & 3.63 \\
\hline $120 \mathrm{mg} / \mathrm{kg}$ EO, challenged & 11.40 & 9.06 & 9.46 & 3.30 \\
\hline $240 \mathrm{mg} / \mathrm{kg}$ EO, challenged & 11.37 & 8.70 & 9.34 & 3.39 \\
\hline SEM & 0.041 & 0.068 & 0.085 & 0.110 \\
\hline \multicolumn{5}{|c|}{ P-value } \\
\hline Challenge & 0.973 & 0.785 & 0.316 & $<0.001$ \\
\hline EO & 0.190 & 0.022 & 0.952 & 0.327 \\
\hline Challenge $\times \mathrm{EO}$ & 0.842 & 0.916 & 0.783 & 0.466 \\
\hline Linear $^{\mathrm{b}}$ & 0.299 & 0.673 & 0.566 & 0.270 \\
\hline Quadratic $^{b}$ & 0.129 & 0.002 & 0.927 & 0.606 \\
\hline
\end{tabular}

${ }^{a}$ Values are means of eight replicates per treatment, and expressed as $\log _{10}$ (copy/g digesta). EO, essential oils; unchallenged, birds without challenge of $C$. perfringens; challenged, birds challenged with C. perfringens from day 14 to 20; SEM, pooled standard error of the mean; Challenge $\times$ EO, interaction between $C$. perfringens challenge and EO supplementation

${ }^{\mathrm{b}}$ When interaction between C. perfringens challenge and EO supplementation was not significant, linear and quadratic polynomial contrasts were performed on the main-effect means to EO dosages 
Table 7 Effect of essential oils on cecal bacterial populations of broilers on day 28

\begin{tabular}{|c|c|c|c|c|}
\hline tem $^{a}$ & Total bacteria & Lactobacillus & Escherichia & C. perfringens \\
\hline \multicolumn{5}{|l|}{ Treatment } \\
\hline Basal diet, unchallenged & 11.18 & 9.14 & 8.83 & 2.68 \\
\hline 60 mg/kg EO, unchallenged & 11.19 & 9.10 & 8.66 & 2.54 \\
\hline 120 mg/kg EO, unchallenged & 11.31 & 9.16 & 8.80 & 2.55 \\
\hline 240 mg/kg EO, unchallenged & 11.00 & 8.99 & 8.55 & 2.42 \\
\hline Basal diet, challenged & 11.18 & 9.22 & 9.24 & 2.79 \\
\hline $60 \mathrm{mg} / \mathrm{kg} \mathrm{EO}$, challenged & 11.24 & 9.30 & 9.01 & 3.24 \\
\hline 120 mg/kg EO, challenged & 10.89 & 9.19 & 8.77 & 2.78 \\
\hline $240 \mathrm{mg} / \mathrm{kg}$ EO, challenged & 10.81 & 9.02 & 8.42 & 2.47 \\
\hline SEM & 0.046 & 0.056 & 0.092 & 0.077 \\
\hline \multicolumn{5}{|c|}{$P$-value } \\
\hline Challenge & 0.126 & 0.473 & 0.428 & 0.078 \\
\hline EO & 0.076 & 0.624 & 0.269 & 0.205 \\
\hline Challenge $\times \mathrm{EO}$ & 0.220 & 0.951 & 0.685 & 0.406 \\
\hline Linear $^{\mathrm{b}}$ & 0.026 & 0.255 & 0.060 & 0.112 \\
\hline Quadratic $^{b}$ & 0.399 & 0.509 & 0.928 & 0.437 \\
\hline
\end{tabular}

Thymol and carvacrol concentrations in intestinal digesta Thymol and carvacrol concentrations in intestinal digesta linearly increased with increased dietary EO dosages $(P<0.001$, Table 8$)$. For birds fed $240 \mathrm{mg} / \mathrm{kg} \mathrm{EO}$, thymol and carvacrol accounted for $60 \mathrm{mg} / \mathrm{kg}$, as each comprised $25 \%$ of the EO product. Their average concentrations in intestinal digesta were 0.80 and $0.71 \mu \mathrm{g} / \mathrm{g}$ (based on digesta wet weight) respectively, which were significantly higher than concentrations assayed in birds fed 60 and $120 \mathrm{mg} / \mathrm{kg}$ EO $(P \leq 0.05)$.

\section{Discussion}

EO and their purified constituents are promising alternatives to growth-promoting antibiotics, and they are generally recognized as safe [6]. Among thousands of EO constituents, thymol and its isomer carvacrol are the main components of commonly used oregano and thyme oil [26]. In the present study, strong antibacterial effects of an EO product and its components thymol and carvacrol were observed in vitro against a panel of pathogenic bacteria, including E. coli, Salmonella, and $C$. perfringens. In broiler chickens challenged with $C$. perfringens, dietary supplementation of thymol and carvacrol-bearing EO did not influence $C$. perfringens numbers. However, EO did alter Escherichia and Lactobacillu populations, and alleviated intestinal lesions in a linear dose-related manner. In addition, thymol and carvacrol concentrations in intestinal digesta were assayed in this study.

Many studies have tested the antibacterial activity of EO, or their constituents, against foodborne pathogens [11, 16, 26-30]. According to Pei et al., MICs of thymol and carvacrol against E. coli were both $400 \mu \mathrm{g} / \mathrm{mL}$ [26]. And Ivanovic et al. have reported that $E$. coli and $S$. Enteritidis are inhibited by thymol at concentrations between 160 and $320 \mu \mathrm{g} / \mathrm{mL}$ [16]. Also, C. perfringens was inhibited effectively by thymol and carvacrol (MICs of 240 and $300 \mu \mathrm{g} / \mathrm{mL}$, respectively) $[29,31]$. In the present

Table 8 Analytical concentrations of thymol and carvacrol in intestinal digesta

\begin{tabular}{|c|c|c|c|c|c|c|c|c|}
\hline \multirow[t]{2}{*}{ Item $^{a}$} & \multicolumn{4}{|c|}{ Dietary EO concentration (mg/kg) } & \multirow[t]{2}{*}{ SEM } & \multirow[t]{2}{*}{$P$-value } & \multirow[t]{2}{*}{ Linear $^{b}$} & \multirow[t]{2}{*}{ Quadratic } \\
\hline & $\overline{0}$ & 60 & 120 & 240 & & & & \\
\hline Thymol ( $\mu \mathrm{g} / \mathrm{g}$ digesta) & ND & $0.21^{\mathrm{B}}$ & $0.46^{\mathrm{B}}$ & $0.80^{\mathrm{A}}$ & 0.076 & $<0.001$ & $<0.001$ & 0.586 \\
\hline Carvacrol ( $\mu \mathrm{g} / \mathrm{g}$ digesta) & ND & $0.20^{\mathrm{B}}$ & $0.29^{B}$ & $0.71^{\mathrm{A}}$ & 0.076 & $<0.001$ & $<0.001$ & 0.677 \\
\hline
\end{tabular}

${ }^{a}$ Values are means of eight replicates per treatment. Means within the same row not sharing a common uppercase superscript letter differ significantly $(P \leq 0.05)$. EO, essential oil contained $25 \%$ thymol and $25 \%$ carvacrol as active components; SEM, pooled standard error of the mean; ND, not detected

${ }^{\mathrm{b}}$ Linear and quadratic responses of intestinal thymol or carvacrol concentrations to dietary EO dosages 
study, strong antibacterial effects of thymol and carvacrol were observed against selected pathogenic bacteria, and similar MICs of thymol and carvacrol were obtained to previous reports. In addition, the E. coli strains we used were more sensitive to thymol, while the $S$. Enteritidis strains we used were more sensitive to carvacrol, compared with other pathogens. Besides, the antibacterial activity of the EO product was comparable to thymol and carvacrol according to the present work, taking into account that the EO product contained $25 \%$ each of thymol and carvacrol as active components.

However, limited information is available regarding EO effects on beneficial probiotic bacteria, such as Lactobacillus. Only slight or no inhibition against Lactobacillus has been reported for carvacrol at $300 \mu \mathrm{g} / \mathrm{mL}$, according to $\mathrm{Si}$ et al. [31]. In the present study, higher concentrations of thymol and carvacrol (up to $6000 \mu \mathrm{g} / \mathrm{mL}$ ) were used to explore their bactericidal activities. The growth of $L$. acidophilus, $L$. reuteri and $L$. salivarius was observed to be inhibited by thymol and carvacrol at an MIC of $1500 \mu \mathrm{g} / \mathrm{mL}$, and be killed at twice the concentration, the $\mathrm{MBC}$. Also, the $\mathrm{EO}$ product was able to kill the tested Lactobacillus strains at $3000 \mu \mathrm{g} / \mathrm{mL}$. Ouwehand et al. have pointed out that L. fermentum and $L$. reuteri growth is inhibited by thymol itself as well as by EO (oregano, rosemary and thyme oils, whose main constitutes were thymol and/or carvacrol) at $500 \mu \mathrm{g} / \mathrm{mL}$ [32]. However, no antibacterial effects against L. plantarum were found for carvacrol even at $3000 \mu \mathrm{g} / \mathrm{mL}$ in Ben Arfa et al.'s study [9]. The contrary results observed here might be explained by differences in the examined Lactobacillus field strains. In addition, the final concentrations of microbial suspensions in the present study were $1-5 \times 10^{5} \mathrm{cfu} / \mathrm{mL}$ for the MIC assays, but a different range of $10^{6}-10^{7} \mathrm{cfu} / \mathrm{mL}$ was employed in Ben Arfa et al.'s study [9]. Nevertheless, the present results showed that Lactobacillus appeared more resistant to $\mathrm{EO}$ and its constitutes than pathogenic bacteria did, which might indicate that intestinal microbiota in animals might be favored under certain concentrations of EO and its constituents.

According to in vitro experiments, additive antibacterial effects between thymol and carvacrol were observed towards all tested bacteria, as the FICI was between 0.5 and 1.0, and FICI was applied to define the nature of interaction. However, FICI values used for the definition of interaction differed between publications, which makes comparison of studies difficult [33]. The definition we adopted in the present study appears to be more acceptable in literature [33]. Based on the definition in the present study, an additive effect (FICI of 0.75 ) between thymol and carvacrol against E. coli also has been observed in previous studies [26, 27]. Similarly, Lambert et al. have reported that thymol and carvacrol in combination show additive effects against Pseudomonas aeruginosa and Staphylococcus aureus using halffold dilutions within a Bioscreen plate [34]. In addition, the additive effects observed here were in agreement with the antibacterial activity of the EO product, whose $\mathrm{MIC}$ and $\mathrm{MBC}$ were comparable to its active components thymol and carvacrol. This would be expected as thymol and carvacrol are isomers with similar chemical structures and likely to have similar mechanisms of antimicrobial activity [35].

In in vivo trial, wheat-based diets were used to favor C. perfringens colonization. However, the challenge did not result in overtly clinical signs of NE or NE-related mortality in the present trial. The deficiency of NetB gene in the $C$. perfringens strain used here might partially explain the loss of characteristic NE, as NetB has been observed to play a major role in NE $[3,36]$. Despite this, C. perfringens challenge damaged intestinal mucosa, as observed by macroscopic lesions, which was similar to previous studies using the same challenge model to create sub-clinical NE $[37,38]$. And dietary EO supplementation alleviated intestinal lesions linearly on day 21 and 28. This was in agreement with previous studies, which have reported that thymol and carvacrol-bearing EO alleviates C. perfringens-induced gut lesions [29, 39].

The abundance of total bacteria, Lactobacillus, Escherichia, and C. perfringens in the gut was estimated using absolute RT-PCR to evaluate group-specific 16s rDNA in extracted community DNA of intestinal digesta. According to Dahiya et al., C. perfringens numbers can reach $10^{7}-10^{9} \mathrm{cfu} / \mathrm{g}$ digesta in typical clinical NE [5], but $C$. perfringens numbers were lower than $10^{4} \mathrm{cfu} / \mathrm{g}$ digesta in the present study. Even though, microbial populations dramatically changed when birds were infected with C. perfringens, with $C$. perfringens populations significantly increased in both ileum and caecum on day 1 post-challenge. These changes were also accompanied by a significant Escherichia increase in ileum. Many populations of bacteria have been reported to coexist with $\mathrm{NE}$ and one of the largest populations is $E$. coli [40]. This was consistent with the results obtained in the current study as well as other work performed by Liu et al. [38].

The results of the present work indicated that dietary supplementation of EO linearly decreased ileal Escherichia counts on day 21 and 28, and linearly decreased the number of total bacteria in caecum on day 28. In addition, Lactobacillus counts were quadratically affected by dietary EO addition in caecum on day 21 . Similarly, Jamroz et al. have reported that the combination of carvacrol, cinnamaldehyde and capsaicin decreases E. coli populations and increase Lactobacillus numbers in broilers fed a wheat-based diet [14]. Also, EO extracted from thyme and anise have been reported 
to decrease $C$. perfringens and E. coli counts in both small and large intestines, accompanied by decreased intestinal lesion score [41]. Changes in intestinal microbiota might be related to alleviation of intestinal lesions with EO supplementation. Interestingly, no inhibition of C. perfringens was observed for thymol and carvacrol in the present study. This was inconsistent with results obtained by Jamroz et al. and Cho et al. regarding the effects of EO on C. perfringens [14, 41]. Notably, in Abildgaard et al.'s study, C. perfringens numbers were not influenced by dietary supplementation of an EO product (containing thymol, eugenol, curcumin, and piperin) [42]. In fact, different from the well-known antibacterial activities of EO in vitro, their in vivo effects on intestinal bacteria are rather limited with variable results. For example, no alteration in intestinal microbiota was observed in broilers fed an EO-supplemented diet according to Cross et al. and Hong et al. [12, 13]. As mentioned above, EO blends containing various constituents have been used in vivo in published reports, making it difficult to compare their efficacy $[6,8,12-15]$.

The antibacterial efficacy of EO is related to the concentrations of active components. However, studies have shown that most EO are absorbed quickly after oral administration. According to Kohlert et al., peak plasma thymol concentration was reached after $2 \mathrm{~h}$ in humans [43]. In piglets, plasma thymol and carvacrol concentrations peaked at 1.39 and $1.35 \mathrm{~h}$, respectively, and thymol and carvacrol were almost completely absorbed in stomach and proximal small intestine [25]. To exert antibacterial activity in vivo, it has been suggested that delivery protection is needed to aid EO in reaching target sites within the gut $[44,45]$. In the present study, the EO product was stabilized with glycerides. Since little information is available regarding the release of active EO components in broiler intestine, thymol and carvacrol concentrations in intestinal digesta were measured in the present study. It was observed that intestinal thymol and carvacrol concentrations increased linearly with increased dietary EO dosages. The highest concentrations observed for thymol and carvacrol were 0.80 and $0.71 \mu \mathrm{g} / \mathrm{g}$, respectively, in broilers fed $240 \mathrm{mg} / \mathrm{kg}$ EO. This was in accordance with the linear inhibition effects of EO on Escherichia in broilers. Similarly, Michiels et al. have investigated thymol and carvacrol concentrations in piglet gastrointestinal digesta [46]. They observed $5 \mu \mathrm{g} / \mathrm{g}$ carvacrol in the proximal small intestine of piglets fed $2 \mathrm{~g} / \mathrm{kg}$ carvacrol diet, and $13 \sim 24 \mu \mathrm{g} / \mathrm{g}$ thy$\mathrm{mol}$ in piglets fed $2 \mathrm{~g} / \mathrm{kg}$ thymol diet [46]. Although the EO used here was coated with glycerides, the analyzed luminal concentrations of thymol and carvacrol were quite low (much lower than their MICs in vitro), which may partially explain the unchanged $C$. perfringens populations in vivo. To our knowledge, this is the first time that in vitro antibacterial activities and luminal availability of active EO constituents in chicken were investigated, providing a link between $\mathrm{EO}$ activities in vitro and their effects in vivo. According to our finding, more efficient protection and release techniques are necessary to improve luminal availability of EO constituents.

Interestingly, some reports have shown that sub-lethal EO concentrations have important effects on bacterial activity $[47,48]$. According to Inamuco et al., at concentrations where growth of Salmonella was not inhibited, carvacrol completely inhibited Salmonella motility, and reduced its invasion of porcine intestinal epithelial cells (47). In another study, carvacrol did not influence the motility of Bacillus cereus, but significantly inhibited toxin production at doses below its MIC [48]. In the present study, intestinal lesions were alleviated linearly with increased EO dosages despite of unaffected C. perfringens populations. These results suggest that thymol and carvacrol might influence $C$. perfringens adhesion to intestinal mucosa or decrease toxin production without affecting its numbers, which need further studies to be verified. Furthermore, previous studies have shown that supplemental EO can enhance cellular and humeral immunity in chickens, which might be involved in affecting intestinal microbiota and gut health $[49,50]$.

\section{Conclusions}

The present study showed that thymol and carvacrol possessed strong antibacterial activity against pathogenic bacterial strains and weak activity towards beneficial Lactobacillus strains in vitro. In addition, an additive effect was found when thymol and carvacrol were applied in combination. In broiler chickens challenged with $C$. perfringens, dietary supplementation of thymol and carvacrol-bearing EO alleviated intestinal lesions, which might be related to changes in intestinal microbiota. More specific studies are required to improve luminal availability of EO constituents, and clarify how EO affect intestinal microbiota in vivo.

\section{Abbreviations \\ cfu: Colony-forming units; DMSO: Dimethylsulfoxide; EO: Essential oils; FIC: Fractional inhibitory concentration; FICl: Fractional inhibitory concentration index; IU: International unit; MBC: Minimal bactericidal concentration; MIC: Minimum inhibitory concentration; MRS: Man-Rogosa- Sharpe; NE: Necrotic enteritis; OD: Optical density; PCR: Polymerase chain reaction; RT-PCR: Quantitative real-time PCR.}

\section{Competing interests}

The authors declare that they have no competing interests.

\section{Authors' contributions}

ED designed the study, carried out the experiments and measurements, and drafted the manuscript. LG, ZL, and WW assisted with the chicken trial and sample collection. DL participated in the paper writing. YG participated in the study's design, coordination and paper writing. All authors read and approved the final manuscript. 


\section{Acknowledgement}

This study was supported by Novus International Company (Missouri, USA) and the earmarked fund for China Agricultural Research Systems (CARS-42).

Received: 2 July 2015 Accepted: 8 December 2015

Published online: 24 December 2015

\section{References}

1. Van Immerseel F, Buck JD, Pasmans F, Huyghebaert G, Haesebrouck F, Ducatelle R. Clostridium perfringens in poultry: an emerging threat for animal and public health. Avian Pathol. 2004:33:537-49.

2. Van Immerseel F, Rood Jl, Moore RJ, Titball RW. Rethinking our understanding of the pathogenesis of necrotic enteritis in chickens. Trends Microbiol. 2009;17:32-6.

3. Keyburn AL, Boyce JD, Vaz P, Bannam TL, Ford ME, Parker D, et al. NetB, a new toxin that is associated with avian necrotic enteritis caused by Clostridium perfringens. PLoS Pathog. 2008;4:e26.

4. Van der Sluis W. Clostridial enteritis is an often underestimated problem. World Poultry Sci J. 2000;16:42-3.

5. Dahiya JP, Wilkie DC, Van Kessel AG, Drew MD. Potential strategies for controlling necrotic enteritis in broiler chickens in post-antibiotic era. Anim Feed Sci Tech. 2006;129:60-88.

6. Brenes A, Roura E. Essential oils in poultry nutrition: Main effects and modes of action. Anim Feed Sci Tech. 2010;158:1-14.

7. Burt S. Essential oils: their antibacterial properties and potential applications in foods - a review. Int J Food Microbiol. 2004:94:223-53.

8. Windisch W, Schedle K, Plitzner C, Kroismayr A. Use of phytogenic products as feed additives for swine and poultry. J Anim Sci. 2008;86:E140-8.

9. Ben Arfa A, Combes S, Preziosi-Belloy L, Gontard N, Chalier P. Antimicrobia activity of carvacrol related to its chemical structure. Lett Appl Microbiol. 2006:43:149-54

10. Kalemba D, Kunicka A. Antibacterial and antifungal properties of essential oils. Curr Med Chem. 2003:10:813-29.

11. Veldhuizen EJA, Tjeerdsma-van Bokhoven JLM, Zweijtzer C, Burt SA, Haagsman HP. Structural requirements for the antimicrobial activity of carvacrol. J Agr Food Chem. 2006;54:1874-9.

12. Cross DE, McDevitt RM, Hillman K, Acamovic T. The effect of herbs and their associated essential oils on performance, dietary digestibility and gut microflora in chickens from 7 to 28 days of age. Brit Poultry Sci. 2007;48:496-506

13. Hong JC, Steiner T, Aufy A, Lien TF. Effects of supplemental essential oil on growth performance, lipid metabolites and immunity, intestinal characteristics, microbiota and carcass traits in broilers. Livest Sci. 2012;144:253-62.

14. Jamroz D, Wiliczkiewicz A, Wertelecki T, Orda J, Skorupińska J. Use of active substances of plant origin in chicken diets based on maize and locally grown cereals. Brit Poultry Sci. 2005;46:485-93.

15. Mathlouthi N, Bouzaienne T, Oueslati I, Recoquillay F, Hamdi M, Urdaci M, et al. Use of rosemary, oregano, and a commercial blend of essential oils in broiler chickens: in vitro antimicrobial activities and effects on growth performance. J Anim Sci. 2012;90:813-23.

16. Ivanovic J, Misic D, Zizovic I, Ristic M. In vitro control of multiplication of some food-associated bacteria by thyme, rosemary and sage isolates. Food Control. 2012;25:110-6.

17. Joray MB, Palacios SM, Carpinella MC. Understanding the interactions between metabolites isolated from Achyrocline satureioides in relation to its antibacterial activity. Phytomedicine. 2013:20:258-61.

18. Ministry of Agriculture of the People's Republic of China. Nutrient requirements of Chinese feeding standard of chicken (GB, NY/T 33-2004). Beijing, China: China Agriculture Press; 2004.

19. Dahiya JP, Hoehler D, Wilkie DC, Van Kessel AG, Drew MD. Dietary glycine concentration affects intestinal Clostridium perfringens and Lactobacilli populations in broiler chickens. Poultry Sci. 2005;84:1875-85.

20. Wise MG, Siragusa GR. Quantitative analysis of the intestinal bacterial community in one- to three-week-old commercially reared broiler chickens fed conventional or antibiotic-free vegetable-based diets. J Appl Microbiol. 2007;102:1138-49.

21. Deplancke B, Vidal O, Ganessunker D, Donovan SM, Mackie RI, Gaskins HR Selective growth of mucolytic bacteria including Clostridium perfringens in a neonatal piglet model of total parenteral nutrition. Am J Clin Nutr. 2002; $76: 1117-25$
22. Malinen E, Kassinen A, Rinttila T, Palva A. Comparison of real-time PCR with SYBR Green I or 5'-nuclease assays and dot-blot hybridization with rDNAtargeted oligonucleotide probes in quantification of selected faecal bacteria. Microbiology. 2003:149:269-77.

23. Steed H, Macfarlane GT, Blackett KL, Macfarlane S, Miller MH, Bahrami B, et al. Bacterial translocation in cirrhosis is not caused by an abnormal small bowel gut microbiota. FEMS Immunol Med Mic. 2011;63:346-54.

24. Lee C, Kim J, Shin SG, Hwang S. Absolute and relative QPCR quantification of plasmid copy number in Escherichia coli. J Biotechnol. 2006;123:273-80.

25. Michiels J, Missotten J, Dierick N, Fremaut D, Maene P, De Smet S. In vitro degradation and in vivo passage kinetics of carvacrol, thymol, eugenol and trans-cinnamaldehyde along the gastrointestinal tract of piglets. J Sci Food Agri. 2008;88:2371-81.

26. Pei RS, Zhou F, Ji BP, Xu J. Evaluation of combined antibacterial effects of eugenol, cinnamaldehyde, thymol, and carvacrol against E. coli with an improved method. J Food Sci. 2009;74:M379-83.

27. Rivas L, McDonnell MJ, Burgess CM, O'Brien M, Navarro-Villa A, Fanning S, et al. Inhibition of verocytotoxigenic Escherichia coli in model broth and rumen systems by carvacrol and thymol. Int J Food Microbiol. 2010;139:70-8.

28. Tepe B, Daferera D, Sökmen M, Polissiou M, Sökmen A. In vitro antimicrobial and antioxidant activities of the essential oils and various extracts of Thymus eigii M. Zohary et P.H. Davis. J Agr Food Chem. 2004;52:1132-7.

29. Timbermont L, Lanckriet A, Dewulf J, Nollet N, Schwarzer K, Haesebrouck F, et al. Control of Clostridium perfringens-induced necrotic enteritis in broilers by target-released butyric acid, fatty acids and essential oils. Avian Pathol. 2010;39:117-21.

30. Zhou F, Ji B, Zhang H, Jiang H, Yang Z, Li J, et al. Synergistic effect of thymol and carvacrol combined with chelators and organic acids against Salmonella Typhimurium. J Food Protect. 2007;70:1704-9.

31. Si W, Ni X, Gong J, Yu H, Tsao R, Han Y, et al. Antimicrobial activity of essential oils and structurally related synthetic food additives towards Clostridium perfringens. J Appl Microbiol. 2009;106:213-20.

32. Ouwehand AC, Tiihonen $\mathrm{K}$, Kettunen $\mathrm{H}$, Peuranen S, Schulze $\mathrm{H}$, Rautonen N. In vitro effects of essential oils on potential pathogens and beneficial members of the normal microbiota. Vet Med-Czech. 2010:55:71-8.

33. Bassole IH, Juliani HR. Essential oils in combination and their antimicrobial properties. Molecules. 2012:17:3989-4006.

34. Lambert RJW, Skandamis PN, Coote PJ, Nychas G-JE. A study of the minimum inhibitory concentration and mode of action of oregano essentia oil, thymol and carvacrol. J Appl Microbiol. 2001;91:453-62.

35. Burt SA, Vlielander R, Haagsman HP, Veldhuizen EJ. Increase in activity of essential oil components carvacrol and thymol against Escherichia coli 0157: H7 by addition of food stabilizers. J Food Prot. 2005;68:919-26.

36. Smyth JA, Martin TG. Disease producing capability of netB positive isolates of $C$. perfringens recovered from normal chickens and a cow, and net $B$ positive and negative isolates from chickens with necrotic enteritis. Vet Microbiol. 2010;146:76-84.

37. Liu D, Guo Y, Wang Z, Yuan J. Exogenous lysozyme influences Clostridium perfringens colonization and intestinal barrier function in broiler chickens. Avian Pathol. 2010:39:17-24.

38. Liu D, Guo S, Guo Y. Xylanase supplementation to a wheat-based diet alleviated the intestinal mucosal barrier impairment of broiler chickens challenged by Clostridium perfringens. Avian Pathol. 2012;41:291-8.

39. Mathis GF, Hofacre C, Scicutella N. Performance improvement with a feed added coated blend of essential oils, a coated blend of organic and inorganic acids with essential oils, or virginiamycin in broilers challenged with Clostridium perfringens. World Poultry Science Association, Proceedings of the 16th European Symposium on Poultry Nutrition, Strasbourg, France, 26-30 August, 2007

40. McReynolds JL, Byrd JA, Anderson RC, Moore RW, Edrington TS, Genovese $\mathrm{KJ}$, et al. Evaluation of immunosuppressants and dietary mechanisms in an experimental disease model for necrotic enteritis. Poultry Sci. 2004:83:1948-52.

41. Cho JH, Kim HJ, Kim $H_{H}$. Effects of phytogenic feed additive on growth performance, digestibility, blood metabolites, intestinal microbiota, meat color and relative organ weight after oral challenge with Clostridium perfringens in broilers. Livest Sci. 2014;160:82-8.

42. Abildgaard L, Hojberg O, Schramm A, Balle KM, Engberg RM. The effect of feeding a commercial essential oil product on Clostridium perfringens numbers in the intestine of broiler chickens measured by real-time PCR 
targeting the a-toxin-encoding gene (plc). Anim Feed Sci Tech. 2010;157:181-9.

43. Kohlert C, Schindler G, März RW, Abel G, Brinkhaus B, Derendorf H, et al. Systemic availability and pharmacokinetics of thymol in humans. J Clin Pharmacol. 2002;42:731-7.

44. De Lange CFM, Pluske J, Gong J, Nyachoti CM. Strategic use of feed ingredients and feed additives to stimulate gut health and development in young pigs. Livest Sci. 2010;134:124-34.

45. Wang Q, Gong J, Huang X, Yu H, Xue F. In vitro evaluation of the activity of microencapsulated carvacrol against Escherichia coli with K88 pili. J Appl Microbiol. 2009;107:1781-8.

46. Michiels J, Missotten J, Van Hoorick A, Ovyn A, Fremaut D, De Smet S, et al. Effects of dose and formulation of carvacrol and thymol on bacteria and some functional traits of the gut in piglets after weaning. Arch Anim Nutr. 2010:64:136-54.

47. Inamuco J, Veenendaal AKJ, Burt SA, Post JA, Tjeerdsma-van Bokhoven JLM, Haagsman HP, et al. Sub-lethal levels of carvacrol reduce Salmonella Typhimurium motility and invasion of porcine epithelial cells. Vet Microbiol. 2012:157:200-7.

48. Ultee A, Smid EJ. Influence of carvacrol on growth and toxin production by Bacillus cereus. Int J Food Microbiol. 2001;64:373-8.

49. Awaad MHH, Elmenawey M, Ahmed KA. Effect of a specific combination of carvacrol, cinnamaldehyde, and Capsicum oleoresin on the growth performance, carcass quality and gut integrity of broiler chickens. Vet World. 2014;7:284-90.

50. Lee SH, Lillehoj HS, Jang SI, Lee KW, Bravo D, Lillehoj EP. Effects of dietary supplementation with phytonutrients on vaccine-stimulated immunity against infection with Eimeria tenella. Vet Parasitol. 2011;181:97-105.

\section{Submit your next manuscript to BioMed Central and we will help you at every step:}

- We accept pre-submission inquiries

- Our selector tool helps you to find the most relevant journal

- We provide round the clock customer support

- Convenient online submission

- Thorough peer review

- Inclusion in PubMed and all major indexing services

- Maximum visibility for your research

Submit your manuscript at www.biomedcentral.com/submit

C) Biomed Central 